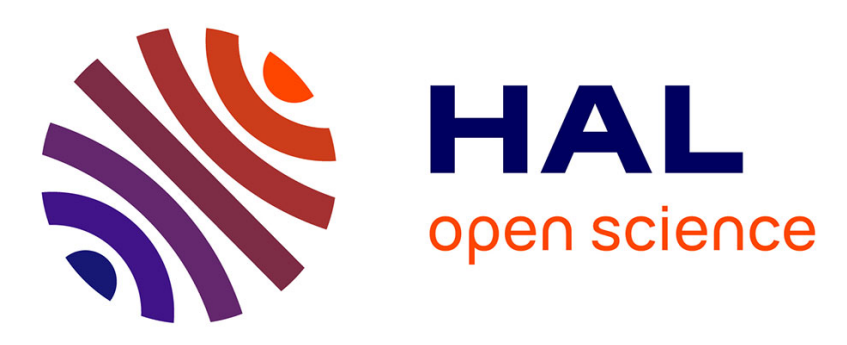

\title{
Caractérisation expérimentale de l'interface fil/matrice dans les composites chanvre/époxy
}

Claire Bonnafous, Fabienne Touchard, Laurence Chocinski-Arnault, Cédric

Dever

\section{- To cite this version:}

Claire Bonnafous, Fabienne Touchard, Laurence Chocinski-Arnault, Cédric Dever. Caractérisation expérimentale de l'interface fil/matrice dans les composites chanvre/époxy. 17èmes Journées Nationales sur les Composites (JNC17), Jun 2011, Poitiers-Futuroscope, France. pp.40. hal-00597912

\section{HAL Id: hal-00597912 \\ https://hal.science/hal-00597912}

Submitted on 2 Jun 2011

HAL is a multi-disciplinary open access archive for the deposit and dissemination of scientific research documents, whether they are published or not. The documents may come from teaching and research institutions in France or abroad, or from public or private research centers.
L'archive ouverte pluridisciplinaire HAL, est destinée au dépôt et à la diffusion de documents scientifiques de niveau recherche, publiés ou non, émanant des établissements d'enseignement et de recherche français ou étrangers, des laboratoires publics ou privés. 


\title{
Caractérisation expérimentale de l'interface fil/matrice dans les composites chanvre/époxy
}

\section{Experimental characterisation of yarn/matrix interface in hemp/epoxy composites}

\author{
Claire Bonnafous ${ }^{1}$, Fabienne Touchard ${ }^{1}$, Laurence Chocinski-Arnault ${ }^{1}$ et Cédric Dever ${ }^{2}$ \\ 1 : Institut Pprime, CNRS-ENSMA-Université de Poitiers UPR 3346, Département Physique et Mécanique des Matériaux \\ ENSMA, 1, avenue Clément Ader, 86961Futuroscope Chasseneuil \\ e-mail : claire.bonnafous@1mpm.ensma.fr, fabienne.touchard@1mpm.ensma.fr, laurence.chocinski@lmpm.ensma.fr \\ 2: VALAGRO : Valorisation des agroressources \\ 86022 POITIERS Cedex \\ e-mail : cdever@valagro-rd.com
}

\begin{abstract}
Résumé
Cette étude propose d'évaluer la liaison fil de chanvre imprégné/résine époxy. Les essais micromécaniques de caractérisation de l'interface fibre/matrice ont été adaptés à une échelle supérieure et associés à des techniques expérimentales permettant la mesure de champs de contrainte et de déplacements. Des composites monofilamentaires contenant un fil de chanvre orienté à $0^{\circ}$ ou $90^{\circ}$ ont été spécifiquement développés. Les essais de fragmentation menés ont permis de définir la contrainte critique de cisaillement interfacial. L'évolution du champ de contrainte suivi par photoélasticimétrie a montré des zones de redistribution des contraintes autour des fragmentations identifiées par des ellipses à $45^{\circ}$ de la direction de sollicitation. Les champs de déformation de points situés dans le fill, dans la résine et à l'interface fil/matrice ont été suivis par des mesures en corrélation d'images numériques réalisées sur la tranche d'éprouvettes monofilamentaires contenant un fil à $90^{\circ}$. Le profil des déformations dans la direction de traction a pu être relié à la structure hélicoïdale du fil. Les résultats ont montré une déformation de cisaillement plus importante pour les points situés à l'interface fil/matrice. Ces mesures ont permis de reconstruire les lois de comportement de points situés dans le fill, dans la résine et à l'interface fil/matrice.
\end{abstract}

\begin{abstract}
In the present work, the link between an impregnated hemp yarn and the epoxy matrix has been investigated. The micromechanical tests usually used to characterize the fibre/matrix interface have been adapted to an upper scale and associated with experimental techniques allowing the measurement of stress and displacement fields. Single yarn composite specimens with yarn axis at $0^{\circ}$ or $90^{\circ}$ to the loading direction have been developed. Fragmentation tests have been performed to determine the critical interfacial shear strength. The changes of stress field during fragmentation process show a stress redistribution identified by four elliptic profiles which occur at $45^{\circ}$ of the tensile loading direction. The build-up of strain field for point measurements in the yarn, in the resin and at the yarn/matrix interface has been tracked by digital image correlation on the edge of single yarn composite specimens with yarn axis at $90^{\circ}$ from the loading direction. The strain profile in the direction of loading can be related to the helicoidal structure of the yarn. Results showed the larger value of shear stresses for points at the yarn/matrix interface. These results allowed to define the behaviour law of points located in the yarn, in the matrix and at the yarn/matrix interface.
\end{abstract}

Mots Clés : Interface, fibres naturelles, fil, corrélation d'images numériques, photoélasticité Keywords : Interface, natural fibres, yarn, digital image correlation, photoelasticity

\section{Introduction}

Différentes études ont caractérisé les composites à renforts longs de chanvre et ont notamment montré le potentiel des tissus de chanvre comme remplacement des tissus de verre dans les composites [1] ainsi que la grande reproductibilité des caractéristiques mécaniques des composites à renforts fils longs de chanvre en dépit de la variabilité détectée sur les fils seuls [2]. Dans notre étude, les renforts utilisés sont des tissus de chanvre. Les composites à renforts tissus présentent une microstructure complexe à laquelle s'ajoute la complexité de la structure des fibres naturelles. Pour 
comprendre le comportement de ces matériaux, deux interfaces peuvent être caractérisées : l'interface fibre/matrice et l'interface fil/matrice. L'interface fibre/matrice a été étudiée dans de nombreux travaux [3-5]. Ce travail propose de caractériser la liaison fil imprégné/résine époxy en adaptant les techniques classiques utilisées pour caractériser l'interface fibre/matrice.

Il existe plusieurs méthodes d'essais micromécaniques de caractérisation de l'interface fibre/matrice permettant d'accéder localement au comportement de l'interface fibre/matrice au moyen d'essais sur des composites modèles monofilamentaires. L'essai de fragmentation est notamment communément utilisé pour caractériser la contrainte critique de cisaillement interfacial dans les composites à fibres naturelles [6-8]. Il consiste à solliciter en traction une éprouvette contenant une fibre courte ou longue noyée dans la résine polymère. Un processus de fragmentation de la fibre est alors initié là où la déformation à rupture de la fibre est dépassée et se poursuit jusqu'à ce que le transfert de charge par cisaillement ne permette plus de générer des contraintes de traction suffisamment importantes pour engendrer une nouvelle fragmentation [9]. Pour comprendre le transfert de charge à l'interface fibre/matrice, les essais sont souvent complétés par des techniques expérimentales permettant d'acquérir les champs de déplacements et de contraintes proches d'hétérogénéités. Ainsi, Wang et al. [10] ont simulé le comportement en fragmentation de composites carbone/époxy en couplant les simulations numériques à une analyse en photoélasticimétrie. Godara et al. [11] ont caractérisé les champs de déformation locaux dans des composites bois/polypropylène grâce à la corrélation d'images numériques en lumière blanche avec une précision de $4 / 100$ de pixel.

Dans ce travail, l'interface fil de chanvre/résine époxy a été caractérisée en réalisant des essais de fragmentation à l'échelle de composites monofilamentaires contenant un fil de chanvre noyé dans la résine époxy. Pour décrire plus précisément la nature du lien entre la matrice et le fil, le champ de contrainte a été déterminé durant le processus de fragmentation grâce à la technique de photoélasticimétrie. Puis, des éprouvettes spécifiques ont été développées pour permettre d'étudier le champ de déformation dans le fil, dans la matrice et à l'interface fil/matrice grâce à la technique de corrélation d'images numériques.

\section{Matériaux et méthodes}

\subsection{Matériaux}

Les fils de chanvre de numéro métrique $\mathrm{Nm} 12$ sont constitués d'un ensemble de fibres enroulées en spirale avec un angle moyen de $11^{\circ}$ mesuré en périphérie du fil (Fig. 1) et un diamètre moyen de $305 \pm 60 \mu \mathrm{m}$ [1]. Un tissu taffetas $4 / 4$ est réalisé avec ces fils et imprégné d'une résine époxy pour former le composite final, un stratifié tissé chanvre/époxy [12]. Les composites monofilamentaires nécessaires pour chaque essai ont été découpés dans des plaques élaborées par l'entreprise Valagro (Poitiers - France). Les fils de chanvre sont alignés dans un moule spécifiquement conçu pour les maintenir durant le processus de fabrication. Les plaques sont alors réalisées par moulage au contact en utilisant de la résine EPOLAM 2022 en respectant une procédure de cuisson optimisée pour obtenir une réticulation complète de la résine $(24 \mathrm{~h}$ à température ambiante, $4 \mathrm{~h}$ à $50^{\circ} \mathrm{C}, 2 \mathrm{~h}$ à $80^{\circ} \mathrm{C}$ et $4 \mathrm{~h}$ à $100^{\circ} \mathrm{C}$ ) [1]. 


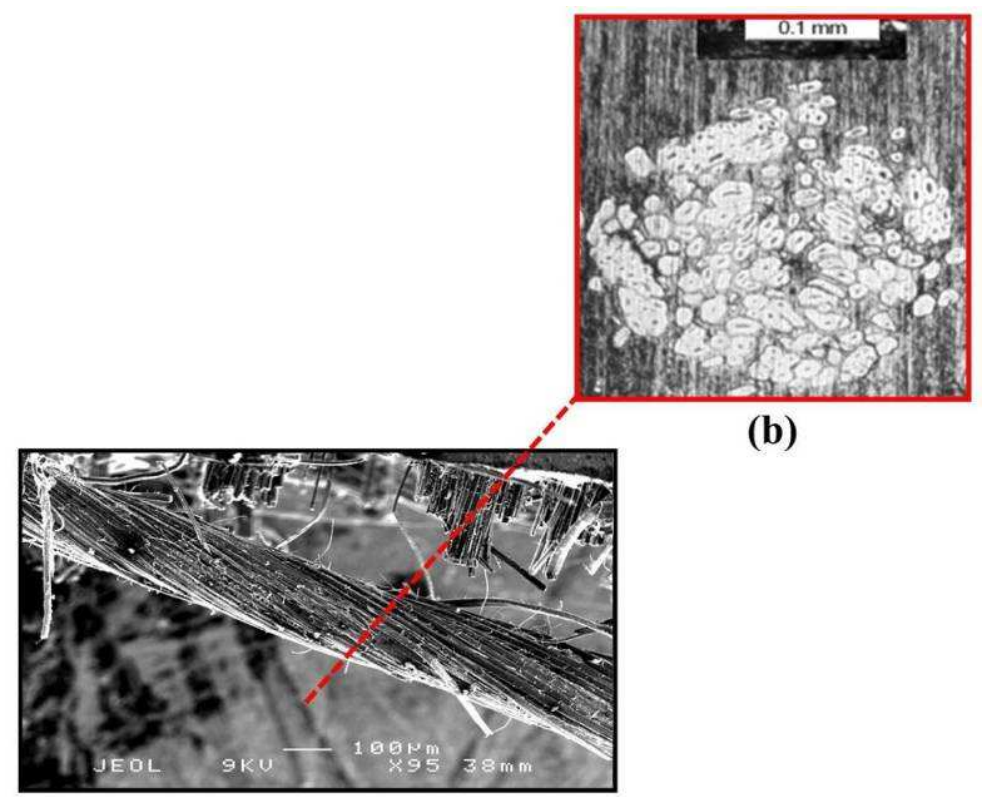

(a)

Fig. 1. Observation en microscopie électronique à balayage d'un fil de chanvre seul (a) et en microscopie optique de la section d'un fil de chanvre imprégné de résine époxy (b).

\subsection{Essai de fragmentation}

Des éprouvettes de résine époxy contenant un seul fil de chanvre orienté à $0^{\circ}$ ont été réalisées (Fig. $2 a$ et $b$ ). Deux géométries d'éprouvettes assurant un état de triaxialité des contraintes suffisant pour initier le processus de fragmentation ont été définies : des éprouvettes haltères notées HAL (Fig. 2a) et des éprouvettes doubles congés notées DC (Fig. 2b).

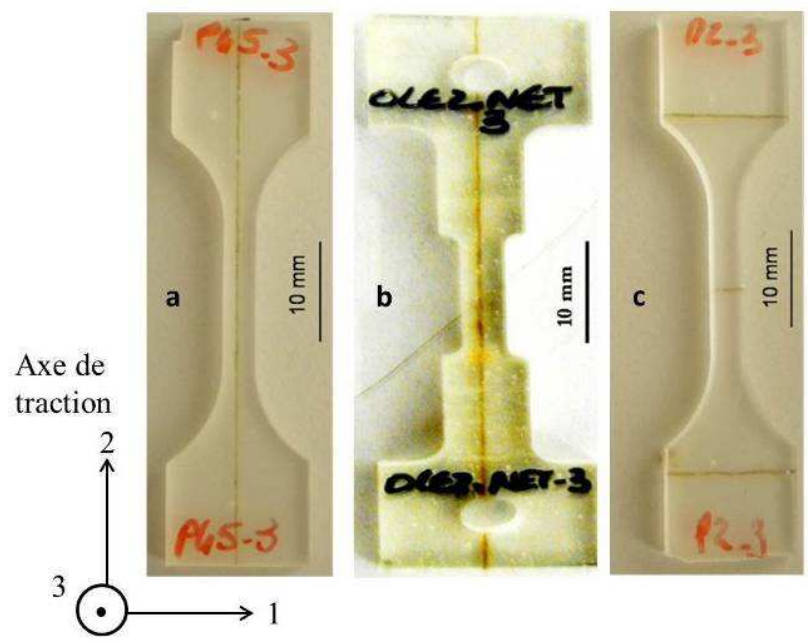

Fig. 2. Géométrie des composites monofilamentaires pour l'essai de fragmentation haltère (a), double congés (b) et pour l'essai de traction transverse au fil (c).

Les essais de fragmentation ont été réalisés sur la machine INSTRON E1000 équipée de mors autoserrants et d'une cellule de force de $1 \mathrm{kN}$ allant en charge statique jusqu'à $750 \mathrm{~N}$. Les essais sont pilotés en vitesse de déplacement de la traverse à $0,5 \mathrm{~mm} / \mathrm{min}$. Les éprouvettes ont été placées dans un polariscope à champ sombre. Une caméra haute résolution $\left(2670 \times 4000\right.$ pixels $\left.^{2}\right)$ focalisée sur la longueur utile de l'éprouvette a été utilisée pour l'acquisition d'images toutes les 2 secondes. Le traitement des images a permis de détecter l'apparition de franges sombres correspondant aux isochromes qui témoignent en temps réel des positions spatiales et temporelles des fragmentations. 


\subsection{Essai de traction transverse au fil}

Pour étudier les champs de déformation au voisinage de l'interface fil/matrice, des éprouvettes de géométrie haltère possédant une orientation du fil à $90^{\circ}$ ont été spécifiquement développées (Fig. 2c). La section du fil sortant sur la tranche de l'éprouvette a été utilisée pour étudier le champ de déformation dans le fil, dans la matrice et à l'interface fil/matrice grâce à la technique de corrélation d'images numériques. Les essais, pilotés en vitesse de déplacement de la traverse à $0,5 \mathrm{~mm} / \mathrm{min}$, ont été réalisés sur la machine INSTRON E1000 équipée de mors autoserrants. Une caméra haute résolution de 2670 x 4000 pixels $^{2}$ placée sur un pied stabilisé autorisant un déplacement dans les trois dimensions au moyen de platines micrométriques acquiert des images de la tranche de l'éprouvette sur laquelle est déposé un mouchetis de grains de 7,5 $\mu \mathrm{m}$. Les vecteurs déplacements de points entre deux images ont été déterminés par comparaison des niveaux de gris des pixels des images en utilisant le logiciel de corrélation appelé Granu développé au laboratoire par Brillaud et al. [13]. Un maillage adaptatif développé tous les $58,8 \mu \mathrm{m}$ permet de suivre le même point physique de la zone d'étude. Pour chaque nœud du maillage, une fenêtre de corrélation de $141 \times 141 \mu \mathrm{m}^{2}$ a été définie pour le calcul des vecteurs de déplacement. Les champs de déformation sont obtenus en utilisant les modules d'intégration et de dérivation du code d'éléments finis ABAQUS.

\section{Résultats et discussion}

\subsection{Analyse du champ de contrainte obtenu par photoélasticimétrie}

La figure 3 présente la visualisation des lignes isochromes, lignes d'égale différence des contraintes principales après chaque fragmentation obtenues par photoélasticimétrie.

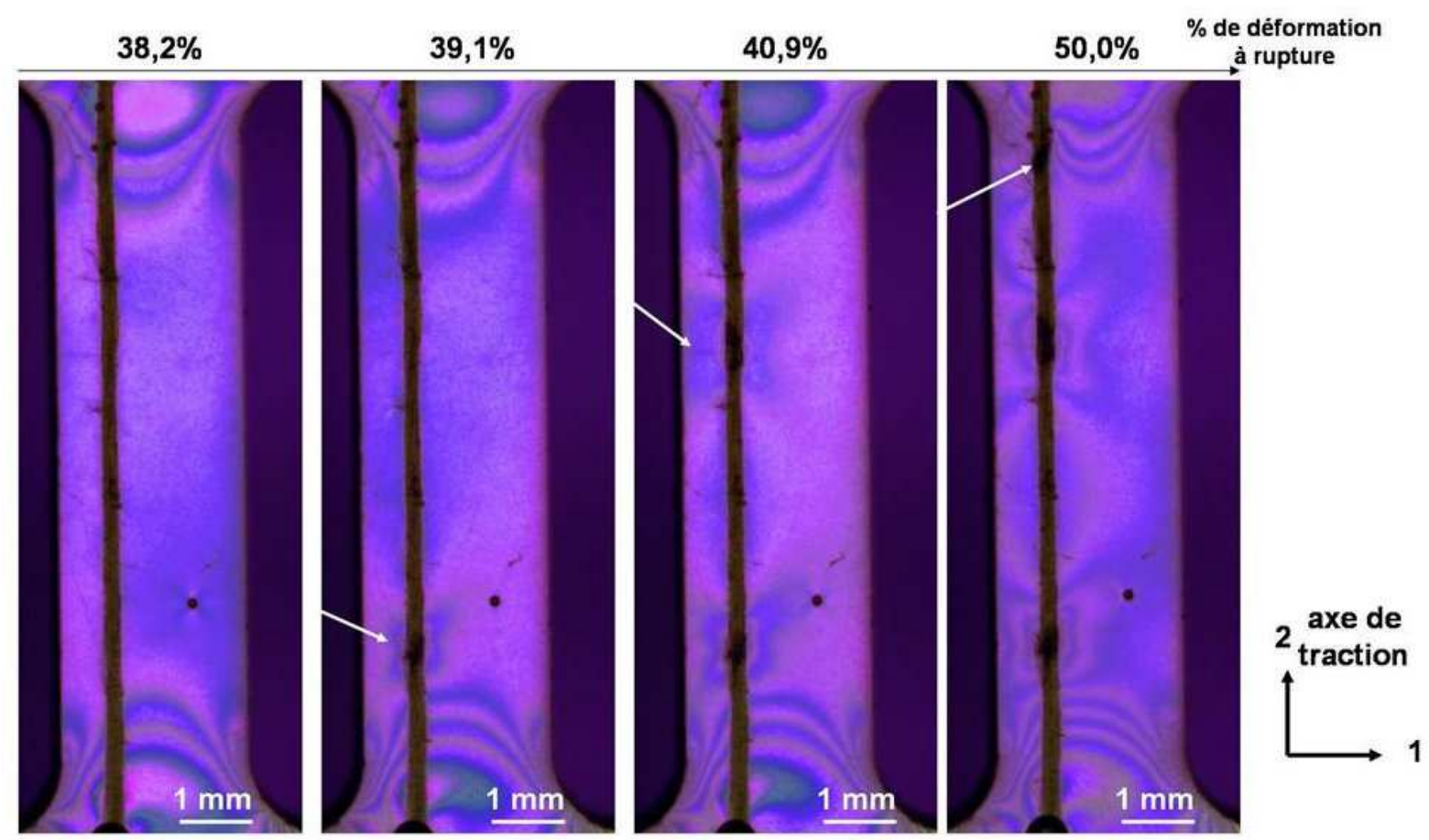

Fig. 3. Visualisation des lignes isochromes après traitement d'images lors du processus de fragmentation d'une éprouvette monofilamentaire chanvre/époxy.

Pour cette éprouvette, les trois premières ruptures du fil ont été détectées successivement à 39,1\%, $40,9 \%$ et $50 \%$ de la déformation à rupture (repérées par les flèches sur la figure 3 ). Avant fragmentation, les isochromes sont symétriques. Lors de la première fragmentation, les ellipses caractéristiques d'une rupture de fil sont observées et une zone de redistribution des contraintes présentant un profil triangulaire apparait dans le reste de l'éprouvette. Après la seconde 
fragmentation, un losange matérialise la redistribution des contraintes entre les deux fragmentations (Fig. 3). A saturation du processus de fragmentation, plusieurs ordres de franges propres au transfert de charge dans la matrice autour des fragmentations sont détectés (Fig. 4).

La figure 4, qui présente la visualisation obtenue en fin d'essai, montre l'évolution et l'apparition d'ordres de frange supérieurs au cours de la traction qui indiqueraient plutôt l'absence de décohésion fil/matrice.

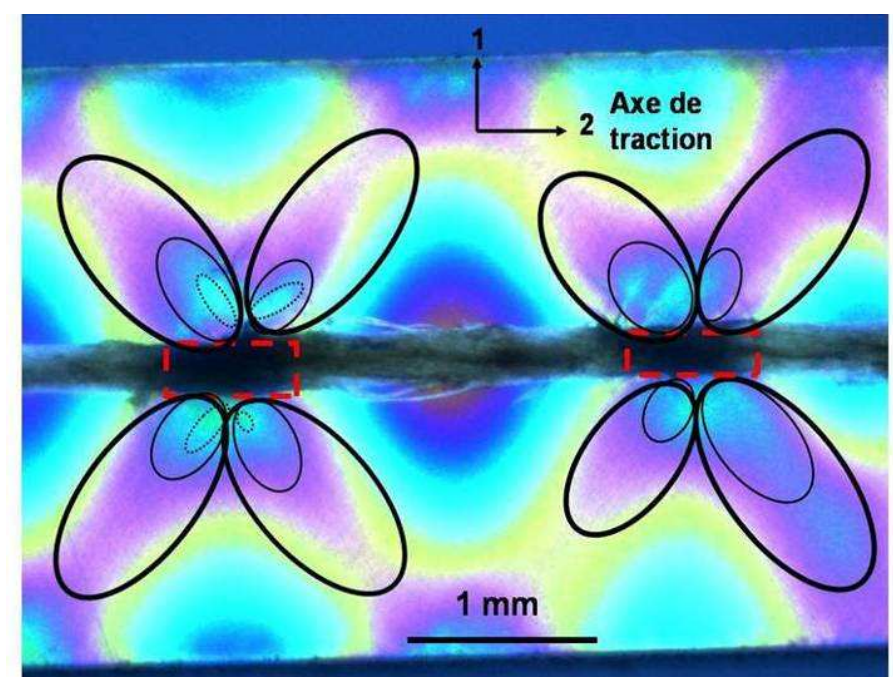

Fig. 4. Visualisation des isochromes proches d'une fragmentation dans une éprouvette monofilamentaire chanvrelépoxy.

La redistribution des contraintes dans la matrice est matérialisée par quatre ellipses identiques représentées en traits pleins ou pointillés (Fig. 4). La zone induite par les ruptures de fibres se situe à $45^{\circ}$ par rapport à la direction du fil, comme l'avaient constaté Wang et al. [10] sur des éprouvettes monofilamentaires carbone/époxy.

Les essais de fragmentation ont été réalisés sur les deux géométries (DC et HAL) pour évaluer la reproductibilité. La figure 5 présente pour chacune des éprouvettes testées, la contrainte $\sigma_{\text {frag }}$ (Fig. 5a) et la déformation $\varepsilon_{\text {frag }}$ (Fig. $5 b$ ) auxquelles survient chaque fragmentation ainsi que les valeurs des déformations et des contraintes à rupture mesurées sur le fil seul et la résine seule [1].
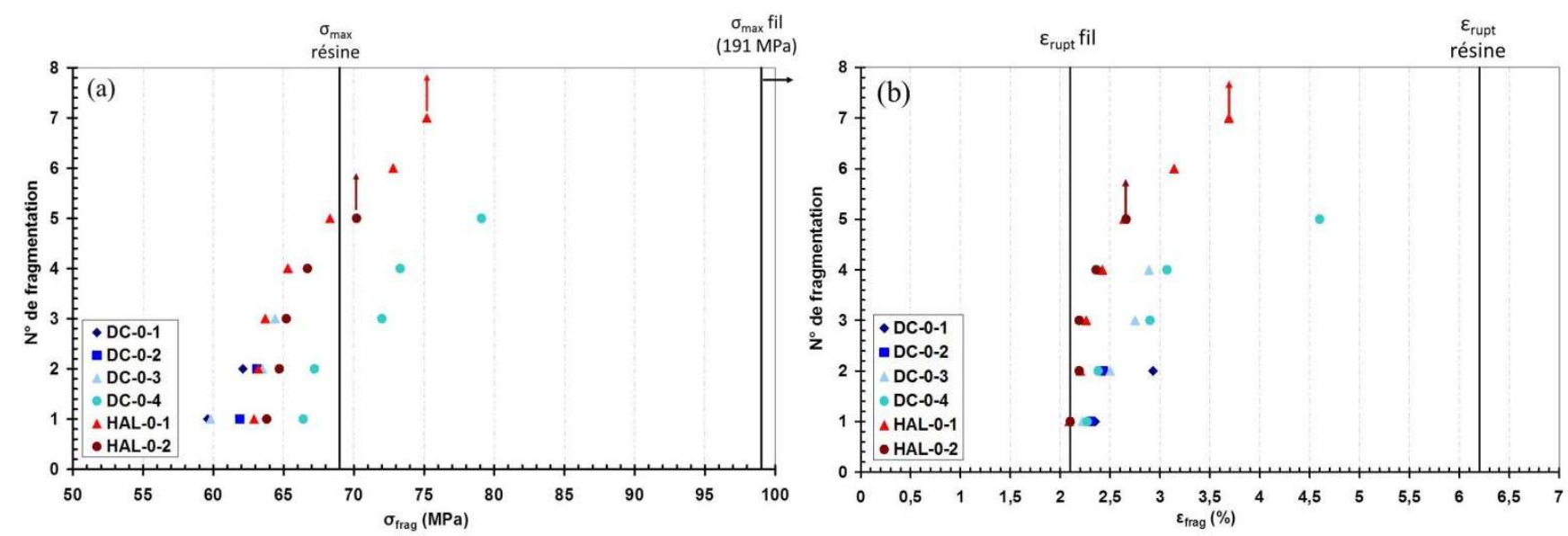

Fig. 5. Contrainte (a) et déformation (b) atteintes à chaque fragmentation du fil lors de l'essai de fragmentation sur éprouvettes monofilamentaires chanvre/époxy.

Les résultats montrent que les valeurs des contraintes et des déformations lors des fragmentations sont assez homogènes même pour des éprouvettes de géométries différentes. Aucun fragment n'est détecté pour une valeur de la déformation inférieure à 2,12\% qui correspond à la déformation à 
rupture du fil de chanvre seul [1]. De la même façon que dans le cas d'un essai de fragmentation sur un composite contenant une fibre unique, le processus de fragmentation est donc initié pour une déformation correspondant à la déformation à rupture du fil seul.

\subsection{Détermination de la contrainte maximale de cisaillement interfacial}

La contrainte critique de cisaillement interfacial notée IFSS peut être déterminée par l'approche en contrainte formulée par Kelly-Tyson [9] (Eq. 1).

$$
\operatorname{IFSS}=\frac{\phi \sigma_{f}\left(l_{C}\right)}{2 l_{C}}
$$

Avec $1_{\mathrm{c}}$ : longueur critique des fragments

$\varnothing$ : diamètre du fil

$\sigma_{\mathrm{f}}\left(l_{\mathrm{c}}\right)$ : contrainte à rupture d'une fibre de longueur $1_{\mathrm{c}}$

La longueur critique de fragmentation $l_{c}$ est obtenue à partir de la longueur moyenne $\bar{l}$ sur l'ensemble des fragments en supposant une distribution symétrique [14]. Le rapport de la longueur critique sur le diamètre est alors défini par l'équation 2 :

$$
\frac{l_{c}}{\phi}=\frac{4}{3}\left(\frac{\bar{l}}{\phi}\right)_{s a t}
$$

La longueur moyenne $\bar{l}$ des fragments du fil pour chaque éprouvette testée a été calculée pour identifier la valeur $\left(\frac{\bar{l}}{\phi}\right)_{\text {sat }}$ pour chaque géométrie d'éprouvette (Fig. 6).

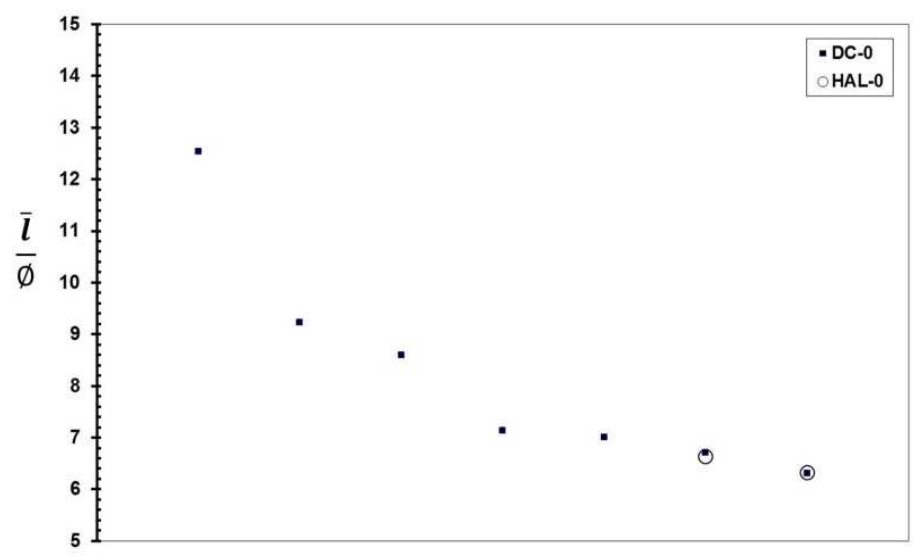

Fig. 6. Rapport de la longueur moyenne des fragments sur le diamètre du fil pour les différentes éprouvettes monofilamentaires chanvrelépoxy testées.

Pour les deux types d'éprouvettes, la valeur à saturation $\left(\frac{\bar{l}}{\phi}\right)_{\text {sat }}$ est voisine de 6 . Ce résultat permet de confirmer que la géométrie de l'éprouvette n'influe pas sur cette valeur à saturation.

La contrainte à rupture du fil $\sigma_{\mathrm{f}}$ à la longueur critique $1_{\mathrm{c}}$ a été extrapolée à partir de la contrainte maximale atteinte par le fil pour des longueurs de fil plus grandes. Pour cela, les travaux de El Asloun et al. [15] ont montré qu'il est possible d'extrapoler la valeur de la contrainte critique d'une fibre $\sigma$ pour des longueurs faibles (Eq. 3). 


$$
L n \sigma=-\frac{1}{m} \ln l+\ln \left[\sigma_{0} \Gamma\left(1+\frac{1}{m}\right)\right]
$$

Avec $m$ et $\sigma_{0}$, les paramètres de Weibull,

$\Gamma$ la fonction Gamma,

et 1 la longueur de fibre testée.

Les données utilisées ainsi que les résultats obtenus sont présentés dans le tableau 1.

\begin{tabular}{|c|c|c|c|c|c|}
\hline \multirow{2}{*}{ Eprouvettes } & \multicolumn{3}{|c|}{ Données d'entrées } & \multicolumn{2}{|c|}{ Grandeurs calculées } \\
\hline & $m_{\infty 0}$ & $\phi(\mu \mathrm{m})$ & $\sigma_{\text {fil }}(\mathrm{MPa})$ & $\sigma_{\mathrm{lc}}(\mathrm{MPa})$ & IFSS (MPa) \\
\hline DC & \multirow{2}{*}{$\begin{array}{c}3,078 \\
{[1]}\end{array}$} & \multirow{2}{*}{$305 \pm 60$} & \multirow{2}{*}{$\begin{array}{c}191 \pm 38 \\
{[1]}\end{array}$} & 354,35 & 21,05 \\
\hline HAL & & & & 354,23 & 21,02 \\
\hline
\end{tabular}

Tab.1. Evaluation des paramètres à rupture de l'interface fil/matrice dans les composites chanvrelépoxy.

Les valeurs trouvées pour la contrainte critique de cisaillement interfacial des deux géométries d'éprouvettes sont très proches. Dans la bibliographie, les différentes valeurs d'IFSS se rapportent à des éprouvettes contenant une fibre unitaire noyée dans la résine époxy. Dans le cas d'une fibre unitaire de lin noyée dans une résine vinylester, la contrainte de cisaillement interfacial est de $24 \pm$ $4 \mathrm{MPa}$, pour une fibre de chanvre noyée dans une résine polypropylène, la valeur de IFSS est de 15,4 $\pm 4 \mathrm{MPa}$. Les mesures expérimentales obtenues ici, effectuées à une échelle différente, sont donc en bonne adéquation avec les valeurs de la bibliographie.

\subsection{Analyse du comportement transverse par corrélation d'images numériques}

Les champs de déformation obtenus par corrélation d'images numériques pour différentes valeurs $\mathrm{du}$ pourcentage de déformation à rupture peuvent être retranscrits sous forme de cartographies enregistrées pour différents pourcentages de la déformation à rupture de l'éprouvette. La figure 7 présente les champs de déformation $\varepsilon_{22}$ sur une éprouvette monofilamentaire chanvre/époxy avec fil à $90^{\circ}$ soumise à une traction transversale à la direction du fil (Fig. 2c). 

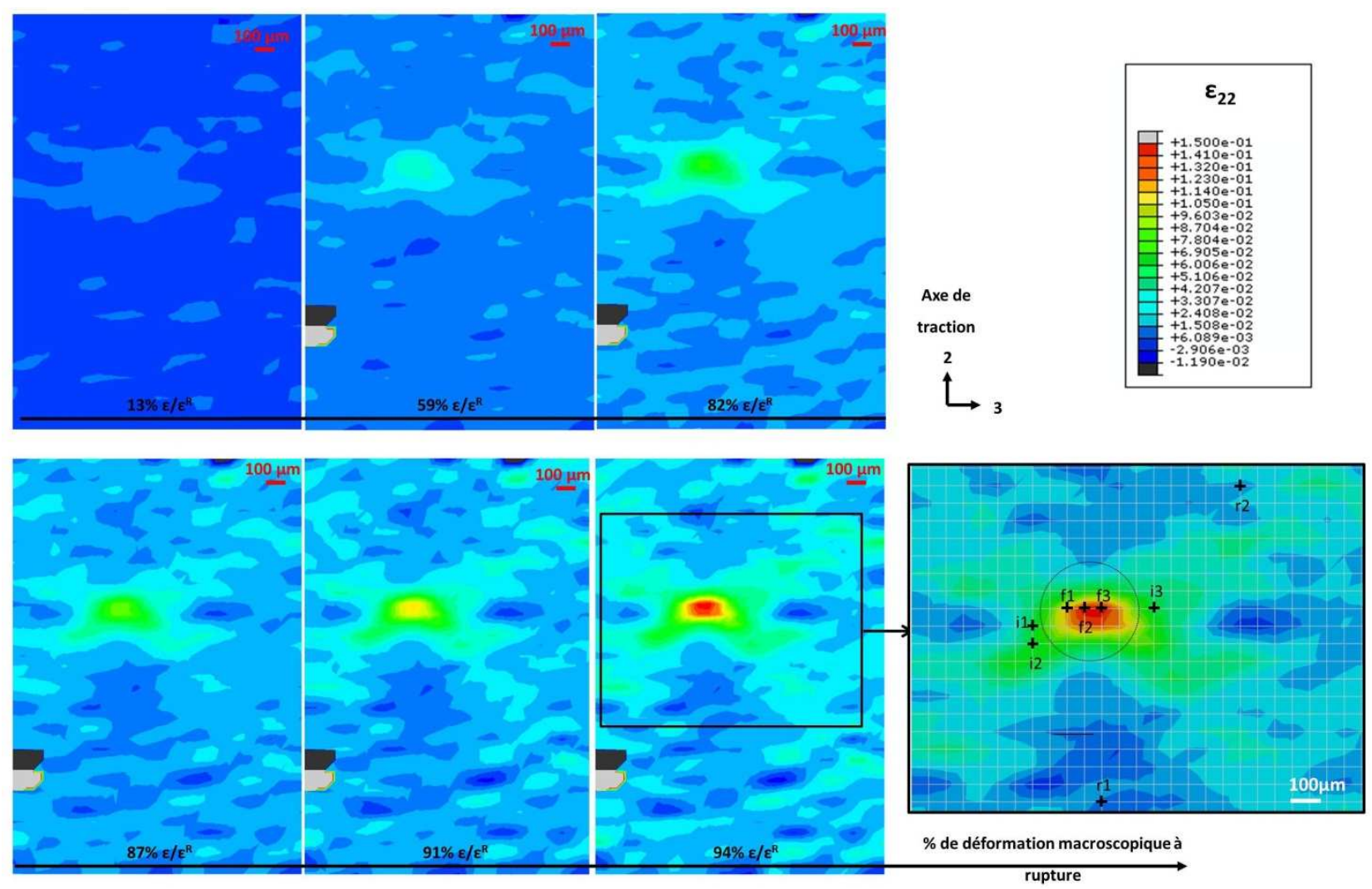

Fig. 7. Cartographies du champ de déformation $\varepsilon_{22}$ mesurées sur la tranche d'une éprouvette haltère monofilamentaire chanvrelépoxy avec fil à $90^{\circ}$ sollicitée en traction, par la technique de corrélation d'images.

Les résultats montrent que la présence du fil est très légèrement visible à $13 \%$ de la déformation à rupture. A partir de 59\% de la déformation à rupture, les ellipses qui matérialisent le transfert de charge dans la matrice sont détectées. Le fil se comporte en réalité comme un concentrateur de déformations au sein de la matrice époxy. La légère dissymétrie du champ de déformation dans le diamètre du fil (Fig. 7) peut être associée au fait que le fil n'est pas parfaitement circulaire. Le gradient de déformation s'accentue quand on augmente le pourcentage de la déformation à rupture. Ce gradient est à mettre en relation avec la structure hélicoïdale du fil, sollicité dans le sens transverse. La plus grande valeur de déformation est détectée au centre du fil. En effet, le fil est constitué d'un ensemble de fibres enroulées en spirale. Lors de la traction, les fibres périphériques sont tirées vers l'extérieur, le processus de rupture du fil dans le sens transverse conduit à une destruction de la structure hélicoïdale du fil et donc à des valeurs de la déformation très importantes (jusqu'à 14,9\% de déformation au cœur du fil alors que le fil ne se déforme que de $2,1 \pm 0,2 \%$ dans le sens longitudinal).

Pour comprendre plus en détail le développement du champ de déformation au sein de l'éprouvette de type haltère, différents points ont été choisis et suivis au cours de l'essai. Deux points ont été choisis dans la matrice $\mathrm{r} 1$ et $\mathrm{r} 2$, trois points au cœur $\mathrm{du}$ fil $\mathrm{f} 1, \mathrm{f} 2$ et $\mathrm{f} 3$ et trois points pour représenter l'interface fil/matrice i1,i2 et i3 (Fig. 7).

En chacun de ces points et à chaque étape de l'essai, les valeurs de déformation mesurées par corrélation d'images et les valeurs correspondantes de la contrainte appliquée à l'éprouvette ont été relevées. Il est ainsi possible de reconstituer le comportement «local» en chacun de ces points au cours de l'essai (Fig. 8). La figure 8 présente ainsi les résultats obtenus pour les trois déformations $\varepsilon_{33}, \varepsilon_{22}, \varepsilon_{32}$ sur la tranche de l'éprouvette. 

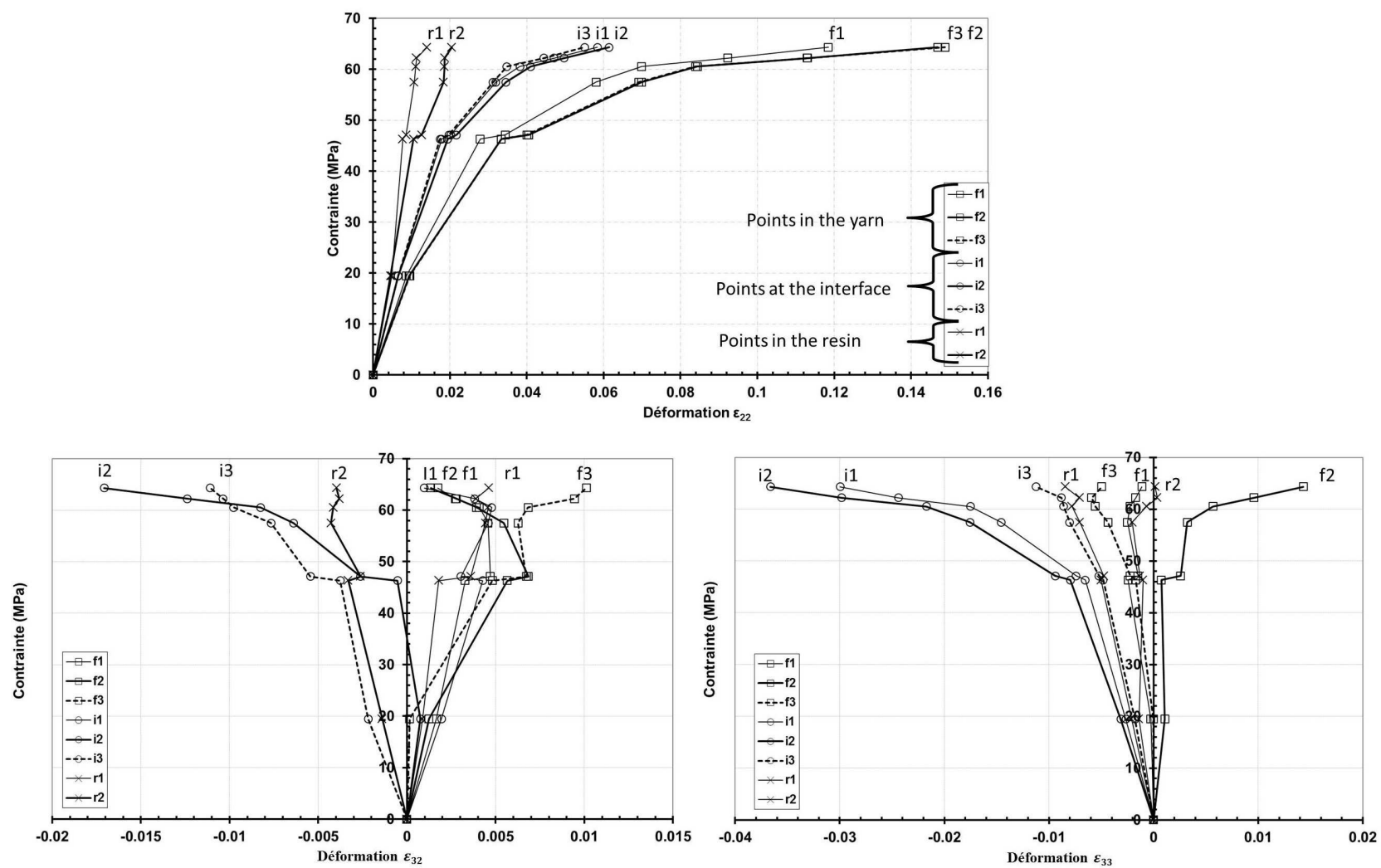

Fig. 8. Suivi, par corrélation d'images, des courbes contrainte macroscopique - déformation locale en différents points au voisinage de l'interface fil/matrice lors d'un essai de traction sur éprouvette monofilamentaire chanvre/époxy à $90^{\circ}$.

Dans la direction de traction, la déformation $\varepsilon_{22}$ la plus importante est détectée dans le fil, le gradient précédemment observé dans le fil est visible grâce aux trois points du fil testés, les points $\mathrm{f} 2$ et $\mathrm{f} 3$ étant très similaires. Les points situés dans l'interface présentent des courbes intermédiaires entre les déformations des points du fil et celles des points de la résine. Les résultats indiquent que la déformation $\varepsilon_{33}$ des points de la résine est négative et proche de celles de certains points du fil. La déformation $\varepsilon_{33}$ négative de la résine s'explique bien par l'effet Poisson. Deux points du fil présentent des valeurs de $\varepsilon_{33}$ proches de celles des points choisis dans la matrice alors qu'un point présente une dilatation. La structure hélicoïdale du fil permet peut être au fil de résister à la compression induite par le coefficient de Poisson de la résine. Les points situés à l'interface fil/matrice présentent de fortes valeurs négatives de $\varepsilon_{33}$ qui seront accentuées par la dilatation de certains points du fil. Les valeurs de la déformation de cisaillement $\varepsilon_{32}$ sont faibles par rapport à celles de $\varepsilon_{22}$ et sont assez bruitées. Cependant, les résultats obtenus montrent bien que ce sont les points situés à l'interface fil/matrice qui subissent les plus fortes déformations de cisaillement, ce qui montre la nécessité d'optimiser sa tenue en cisaillement.

\section{Conclusion}

L'optimisation de l'interface fibre/matrice dans les composites à renforts de fibres naturelles est nécessaire pour permettre aux fibres naturelles de remplacer les fibres synthétiques dans les matériaux composites. Dans le cas des composites à renforts tissés, la compréhension des mécanismes de liaison fil et fibre/matrice est nécessaire. En première approche, l'un des indicateurs de la qualité de l'interface est la contrainte critique de cisaillement interfacial. L'étude expérimentale réalisée s'appuie sur des essais de fragmentation transposés à une échelle différente pour évaluer la liaison fil imprégné/matrice. L'utilisation de la technique de photoélasticimétrie permet l'observation de plusieurs ordres de franges isochromes qui caractérisent le transfert de charge dans la matrice à $45^{\circ}$ par rapport à la direction de sollicitation. Cette compréhension a été 
renforcée par des mesures par corrélation d'images numériques réalisées sur la tranche d'éprouvettes monofilamentaires à $90^{\circ}$. Les lois de comportement de points situés dans le fil, dans la résine et à l'interface fil/matrice ont ainsi pu être déterminées expérimentalement. Les techniques développées dans cette étude, notamment la photoélasticimétrie et la mesure des déformations par intercorrélation d'images numériques ont permis d'apporter des réponses à la problématique de caractérisation de la liaison fil imprégné/matrice.

\section{Remerciements}

Les auteurs remercient la Région Poitou- Charentes pour son soutien financier.

\section{Références}

[1] C. BONNAFOUS, « Analyse multi échelle des mécanismes d'endommagement de composites chanvre/époxy à renforts tissés. Caractérisation de l'interface fibre/matrice ». PhD thesis, ENSMA-Poitiers 2010.

[2] B. MADSEN, P. HOFFMEYER, H. LILHOLT, « Hemp yarn reinforced composites - II. Tensile properties ». Composites Part A: Applied Science and Manufacturing, Vol. 38, pp. 2204-2215, 2007.

[3] C. BALEY, B. LAMY, « Caractérisation des fibres de lin. Application à l'optimisation de composites fibres de linrésine époxy ». JST AMAC-Compiègne, 1999.

[4] L. MWAIKAMBO, M. ANSELL, « Mechanical properties of alkali treated plant fibres and their potential as reinforcement materials. I. hemp fibres ». Journal of Materials Science, Vol. 41, pp. 2483-2496, 2006.

[5] J. PARK, S.T. QUAMG, B. HWANG, et K.L. DEVRIES, « Interfacial evaluation of modified Jute and Hemp fibers/polypropylene (PP)-maleic anhydride polypropylene copolymers (PP-MAPP) composites using micromechanical technique and nondestructive acoustic emission ». Composites Science and Technology, Vol. 66, pp. 2686-2699, 2006.

[6] G. BECKERMANN, K. PICKERING, « Engineering and evaluation of hemp fibre reinforced polypropylene composites: Micro-mechanics and strength prediction modelling ». Composites Part A: Applied Science and Manufacturing, Vol. 40, pp. 210-217, 2009.

[7] R. JOFFE, J. ANDERSONS, L. WALLSTRÖM, « Strength and adhesion characteristics of elementary flax fibres with different surface treatments ». Composites Part A: Applied Science and Manufacturing, Vol. 34, pp.603-612, 2003.

[8] J. ANDERSONS, R. JOFFE, M. HOJO, S. OCHIAI, « Fibre fragment distribution in a single-fibre composite tension test ». Composites Part B: Engineering, Vol. 32, pp. 323-332, 2001.

[9] A. KELLY, W. TYSON, « Tensile properties of fibre-reinforced metals: Copper/tungsten and copper/molybdenum ». Journal of the Mechanics and Physics of Solids, Vol. 13, pp. 329-350, 1965.

[10] X. WANG, B. ZHANG, S. DU, Y. WU, et X. SUN, « Numerical simulation of the fiber fragmentation process in single-fiber composites ». Materials \& Design, Vol. 31, pp. 2464-2470, 2010.

[11] A. GODARA, D. RAABE, I. BERGNANN, R. PUTZ, U. MÜLLER, «Influence of additives on the global mechanical behavior and the microscopic strain localization in wood reinforced polypropylene composites during tensile deformation investigated using digital image correlation ». Composites Science and Technology, Vol. 69, pp.139-146, 2009.

[12] C. BONNAFOUS, F. TOUCHARD, L. CHOCINSKI-ARNAULT, « Damage mechanisms in hemp-fibre woven fabric composites studied by acoustic emission ». 17th International Conference on Composite Materials Proceedings, (ICCM. 17), Edinburgh, UK, 2010.

[13] J. BRILLAUD, F. LAGATTU-TOUCHARD, « Limits and possibilities of laser speckle and white light image correlation methods : teory and experiments ». Applied Optics, Vol. 41, pp. 6603-6613, 2002.

[14] M.C. PAIVA, C.A. BERNARDO, M. NARDIN, « Mechanical, surface and interfacial characterisation of pitch and PAN-based carbon fibres ». Carbon, Vol. 38, pp. 1323-1337, 2000.

[15] M. El ASLOUN, J. DONNET, G. GUILPAIN, M. NARDIN, J. SCHULTZ, « On the estimation of the tensile strength of carbon fibres at short lengths ». Journal of Materials Science, Vol. 24, pp. 3504-3510, 1989. 